\title{
Genetic and environmental effects in paroxysmal nocturnal hemoglobinuria: this little PIG-A goes "Why? Why? Why?"
}

\author{
Neal S. Young and Jaroslaw P. Maciejewski \\ Hematology Branch, National Heart, Lung, and Blood Institute, NIH, Bethesda, Maryland, USA \\ Address correspondence to: Neal S. Young, National Heart, Lung, and Blood Institute, \\ NIH, Building 10, Room 7C-103, Bethesda, Maryland 20892-1652, USA. \\ Phone: (301) 496-8396; Fax: (301) 496-8396; E-mail: youngn@gwgate.nhlbi.nih.gov.
}

Paroxysmal nocturnal hemoglobinuria $(\mathrm{PNH})$ has been a medical curiosity since the description of the first case in the mid-19th century by the English physician Sir William Gull. (See Rosse [ref. 1] for an eloquent history of studies on this disease.) Gull's patient suffered morning crises of "intermittent hematinuria," which was later recognized as the destruction of red cells in circulation. Intravascular hemolysis is unusual, as erythrocyte destruction occurs in the reticuloendothelial system in most hemolytic anemias. $\mathrm{PNH}$ was puzzling in the laboratory as well: red cells were lysed in the presence of acidified serum by a factor that was not an antibody. When this property was utilized to formulate the diagnostic Ham's test, the symptoms and signs of the disease were broadened, and $\mathrm{PNH}$ was more fully characterized by a clinical triad of hemolysis, venous thrombosis, and marrow failure. Whereas thrombotic disease, which is often in unusual anatomical locations, recurrent, and resistant to therapy, is the major cause of death among Western patients, pancytopenia predominates as a manifestation of PNH in the Far East. PNH is closely related to aplastic anemia (AA), in which an autoimmune reaction leads to hematopoietic cell destruction and an empty bone marrow (2). AA usually responds to immunosuppressive therapies (3). Occasional patients who have been successfully treated with immunosuppressive drugs later manifest symptoms of $\mathrm{PNH}$, evidently as a result of clonal expansion of cells prone to hemolysis and thrombosis. Furthermore, since PNH clones can be detected in as many as $25-35 \%$ of AA patients at presentation, the late outgrowth of these clones may not necessarily be seen as a complication of the immunosuppression, but rather as a consequence of persistent low-level PNH that predated and possibly contributed to the development of AA.

Despite its rarity, PNH has been studied by exceptional clinical and laboratory investigators, every era attracting a cohort of scientists to concentrate on a different aspect of the disease. Perhaps surprisingly, these often disparate lines of research led to the unraveling of many of PNH's mysteries (Figure 1) in logical sequence. The emphasis in the early 20 th century was the mechanism for red cell lysis by treated sera, shown to be activation of the alternative pathway of complement (4). The erythrocyte defect was identified functionally as an inability to inactivate complement on the cell surface, in turn due to a deficit in specific membrane proteins (decay-accelerating factor [DAF, or CD55] and membrane inhibitor of reactive lysis [MIRL, or CD59]). Not only were these proteins absent, but PNH cells lacked a variety of others, including leukocyte alkaline phosphatase and erythrocyte acetylcholinesterase. Ultimately, demonstration of the enzymatic release of alkaline phosphatase by a specific phospholipase led to the identification of a structural feature common to this functionally diverse group of proteins: a distinctive biochemical linkage to the cell membrane, the glycosylphosphatidylinositol (GPI) anchor. Preformed GPI is attached covalently to proteins of appropriate carboxyl sequence and links them to the plasma membrane through the phosphatidylinositol moiety. GPI-anchored proteins thus lack the cytosolic tail of the more common transmembrane cell surface proteins, and they also appear to cluster on the cell surface in biophysically distinctive regions termed "rafts." Almost 100 different GPI-anchored proteins, including enzymes, adhesion molecules, receptors, and blood group anti- gens, have been identified to date in mammalian cells (5). The GPI anchor is evolutionarily conserved, and in parasites, GPI-anchored proteins predominate on the cell surface (6).

The biosynthesis of the GPI anchor was determined for trypanosomes and later in deficient mammalian cell lines (7). The genetic defect in PNH, discovered by expression cloning and then phenotypic correction of mutant cell lines, was localized to the X-linked phosphatidylinositol glycan class A (PIG-A) gene (8), whose product is required for the transfer of $N$-acetylglucosamine to phosphatidylinositol, an early step in the synthesis of the GPI anchor (4). Immortalized cell lines from PNH patients all belong to the class A complementation group; the most frequent $P I G-A$ alterations are small deletions that create stop codons or frameshifts, followed by missense mutations and small insertions.

PIG-A mutations in PNH are somatic, occurring in hematopoietic stem cells. Although there are germline polymorphisms in PIG-A, a constitutional form of $\mathrm{PNH}$ has not been described, and knockout animals have all been chimeras. Most likely, although the defect is compatible with survival and function of adult hematopoietic cells, embryonal development cannot proceed without GPI-anchored proteins. PIG- $A$ mutations in all cases represent loss-of-function alleles and so would be expected to be recessive. Nevertheless, the disease phenotype is seen not only in men, who are hemizygous for the gene, but also, and with equal prevalence, in women. Due to random inactivation of the $\mathrm{X}$-chromosome in the hematopoietic system, each blood cell in a female carries only a single transcriptionally active copy of PIG-A. A given stem cell and its progeny will 
express the PNH phenotype only if their active allele is mutated, but the hemolytic and thrombotic phenotype is evident despite the presence of some normal cells. The clonal origin of PNH cells was first inferred from G6PD enzyme analysis of circulating red cells in informative heterozygous females. All defective erythrocytes, leukocytes, and platelets are derived from a single affected hematopoietic progenitor cell carrying a particular PIG-A mutation. However, in some patients, genetic analysis reveals multiple PNH clones, which may persist for many years (9). Healthy individuals also harbor tiny PNH clones. Lymphocytes of PNH phenotype and PIG-A genotype have appeared in lymphoma patients treated with Campath-1, a monoclonal antibody that coincidentally recognizes a GPI-anchored protein (10), and very small numbers (20-60 per million) of GPI-anchored protein-deficient, $P I G-A^{-}$ granulocytes have been detected in the blood of normal persons (11).

\section{Models of PNH development}

The simplest model to explain how somatic mutations in PIG-A account for the development of PNH predicts that PIG- $A^{-}$cells enjoy an intrinsic advantage over PIG- $A^{+}$cells, resulting in expansion of the defective clone (Figure 2a). Surprisingly, however, clinical observations and much experimental data do not support this hypothesis. Most obviously, PNH does not behave like a leukemia, with inexorable proliferation of abnormal cells. Rather, in most patients, the proportion of PNH cells is stable over years and their differentiation is normal. The presence of PIG- $A^{-}$cells in normal adults is also difficult to reconcile with this model, given the rarity of the disease. In chimeric knockout mice, $P I G-A^{-}$ cells also constitute a minor, static fraction of the marrow or circulating hematopoietic compartments, and PIG$A^{-}$and normal cells behave similarly in paired tissue culture assays (12-14). In most laboratories, PNH patient bone marrow or blood, while often globally deficient in hematopoietic progenitor numbers, has not shown unanticipated outgrowth of either the PIG-A- or normal phenotype cells in colony-forming or long-term culture assays that would indicate a functional difference (15-18). Only one group has reported a major difference between PIG- $A^{-}$cells and controls in susceptibility to apoptosis, but

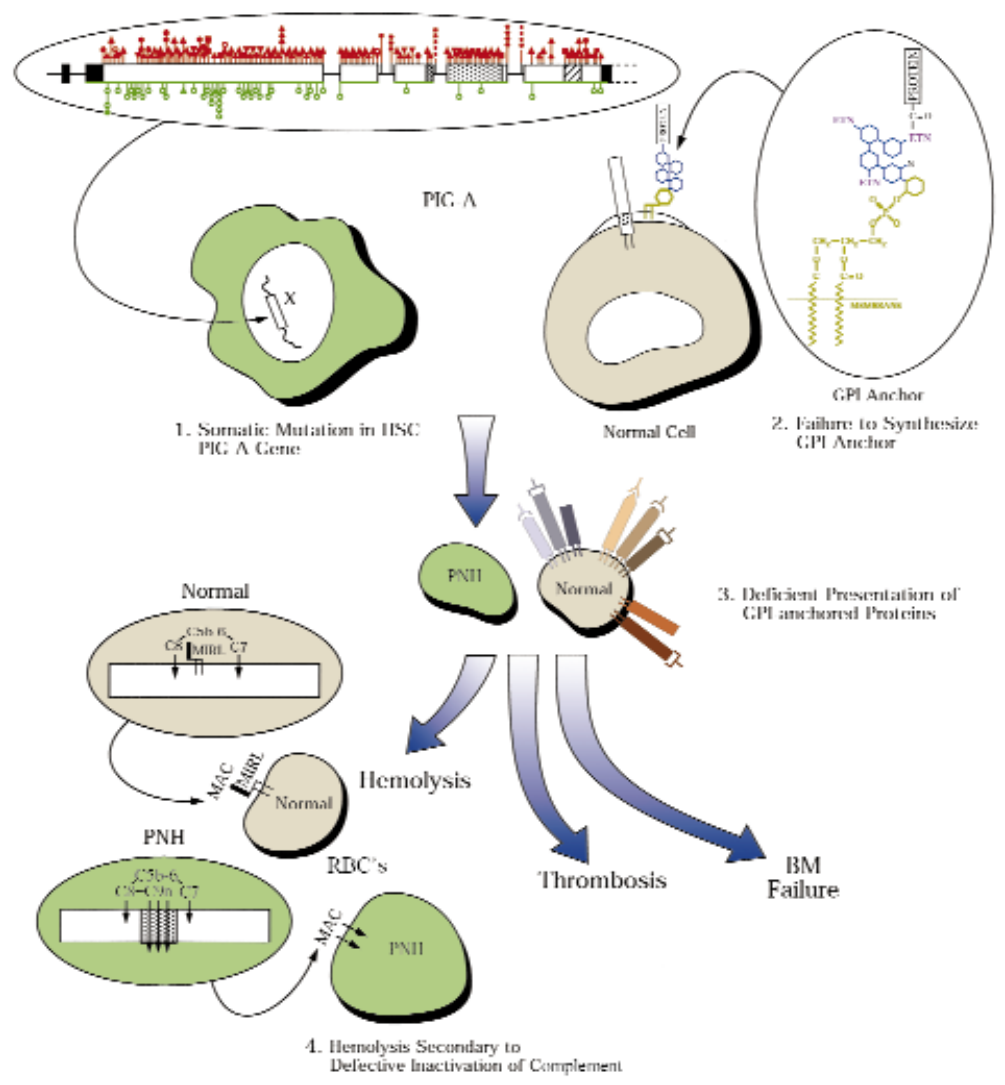

Figure 1

The pathophysiology of PNH. The genetic basis of the disease is diagrammed at upper left. Somatic mutations in the X-linked PIG-A gene occur in a hematopoietic stem cell. PIG-A consists of six exons with an open reading frame of $1455 \mathrm{bp}$; the putative protein product has 484 amino acids and an estimated molecular weight of $60 \mathrm{kDa}$. Small deletion and insertion mutations (dark triangles) are most common in $\mathrm{PNH}$ and are distributed throughout the coding sequence; missense mutations (dark circles) appear to cluster in exon 2, suggesting that this region encodes for a functionally crucial portion of the protein (see ref. 29 for details of the GPI-anchor chemical structure). At upper right, GPI-anchored proteins and conventional transmembrane proteins are contrasted, and the structure of the GPI anchor is shown in the inset. The anchor is a glycolipid composed of the lipid phosphatidylinositol, the sugars $N$-glucosamine and mannose, and ethanolamine. The acyl/alkylglycerol of the lipid anchors the structure in the bilayer of the cell membrane; an ethanolamine links the carboxyl terminal amino acid of the protein to the oligosaccharide portion of the anchor. The PIG-A gene product contributes to the $\mathrm{N}$-acetyl-glucosaminyl transferase activity that adds this sugar to inositol, an early step in construction of the anchor; the entire anchor structure is preformed and then covalently bound to the protein in a transamidation reaction in the endoplasmic reticulum (not shown). Expansion of the PIG-A- clone is required for PNH (see text and Figure 2). Absence of GPIanchored proteins, especially CD59 (MIRL), accounts for hemolysis; the inset shows the role of MIRL in blocking the late complement attack complex, by first binding to $\mathrm{C} 8$ and inhibiting spontaneous binding of $\mathrm{C} 9$ and then preventing $\mathrm{C} 9$ polymerization and subsequent red cell lysis. BM, bone marrow; RBCs, red blood cells; MAC, membrane attack complex.

this finding has not been reproduced in other laboratories (ref. 19 and our unpublished data). Furthermore, there is no known molecular mechanism for GPI-anchored proteins to influence programmed cell death.

A second class of models to explain the etiology of PNH holds that the PIG-A defect must be combined with some extrinsic factor to promote clonal expansion (Figure $2 \mathrm{~b}$ ). The frequent association of PNH with AA first sug- gested that the hypocellular failed marrow might be such a permissive environment $(20,21)$. However, while PNH clones are present in a very large proportion of AA patients (about $20 \%$ in our recent report; ref. 22), PNH does not occur after chemotherapy or radiationinduced aplasia, following stem cell transplant, or in constitutional Fanconi's anemia (22). The presence of PNH does appear to correlate with immunologically mediated marrow failure, and 
is a positive predictor of response to immunosuppressive therapy in AA and myelodysplasia. In addition, $\mathrm{PNH}$ is strongly associated with the MHC haplotype HLA-DR2 (our unpublished data). This MHC association might indicate that an interaction between certain GPI-anchored proteins and the immune system is crucial for $\mathrm{PNH}$ pathogenesis. For example (see Figure 2e), the absence of these proteins in certain genetic backgrounds might allow PNH cells to escape immune surveillance. Indeed, a number of mammalian GPI-anchored proteins serve immune functions or act as ligands for T-cell receptors. Work with one inhibitory T-cell clone derived from an AA patient showed that antibodies to the GPI-anchored CD59 protein, which may interact with the T-cell surface protein CD2, appear to block killing of lymphoblastoid cell targets (23). In PNH, loss of CD59 could result in less efficient killing of GPI-deficient hematopoietic target cells by putative autoimmune Tcell clones. Similar mechanisms could also affect natural killer-mediated cell killing; for example, we described a GPIanchor protein-deficient Ramos cell line that was extremely resistant to natural killer cell lysis, but the PIG-A gene in these cells was normal (24). Recently, an interesting mechanism was described for proinflammatory cytokine production after heat shock protein stimulation through CD14 (25), suggesting that lack of certain GPI-anchored proteins may locally lead to impaired intra- and extracellular signaling. Nevertheless, extensive efforts (many of them unreported) by several laboratories to determine a difference between normal and PIG- $A^{-}$target cells in a wide variety of immunologic assays have been unsuccessful (26). In our unpublished studies, for example, using paired normal and PIG- $A^{-}$cell lines and primary clinical samples as targets, we have been unable to document differences in susceptibility to autologous and allogeneic T-cell cytotoxicity or ability to activate T cells, or in their proliferation after challenge with cytokines or Fas agonists.

\section{PNH as a consequence of poor cell growth?}

In this issue of the JCI, Chen and colleagues identify a major difference in in vitro proliferation between $P I G-A^{-}$and PIG- $A^{+}$CD 34 cells taken from the marrow of PNH patients (27). Remarkably, this difference appears to represent defi- ciency among the "normal" cells in these individuals, rather than an advantage for the PIG-A mutant cells. When a few hundred CD34 cells, sorted by the presence or absence of CD59 to identify normal and $\mathrm{PNH}$ populations, were cultured in the presence of growth factors, the cells with the PNH phenotype behaved normally, producing several hundred thousand progeny, while CD34 cells of normal phenotype from $\mathrm{PNH}$ patients showed little growth. This spectacular difference stands in contrast to most previous studies of whole marrow and CD34 cells using standard colony and long-term marrow cultures - in which bone marrow cells derived from $\mathrm{PNH}$ patients give rise to progeny of both normal and mutant phenotypes. No methodologic explanation for this discrepancy is evident. However, a similar result has been observed in immunodeficient mice that were infused with bone marrow from $\mathrm{PNH}$ patients; in this experiment, the abnormal clone engrafted but little or no normal human hematopoiesis could be detected (28).

The suggestion that loss of PIG-A expression restores normal proliferation to otherwise poorly growing or
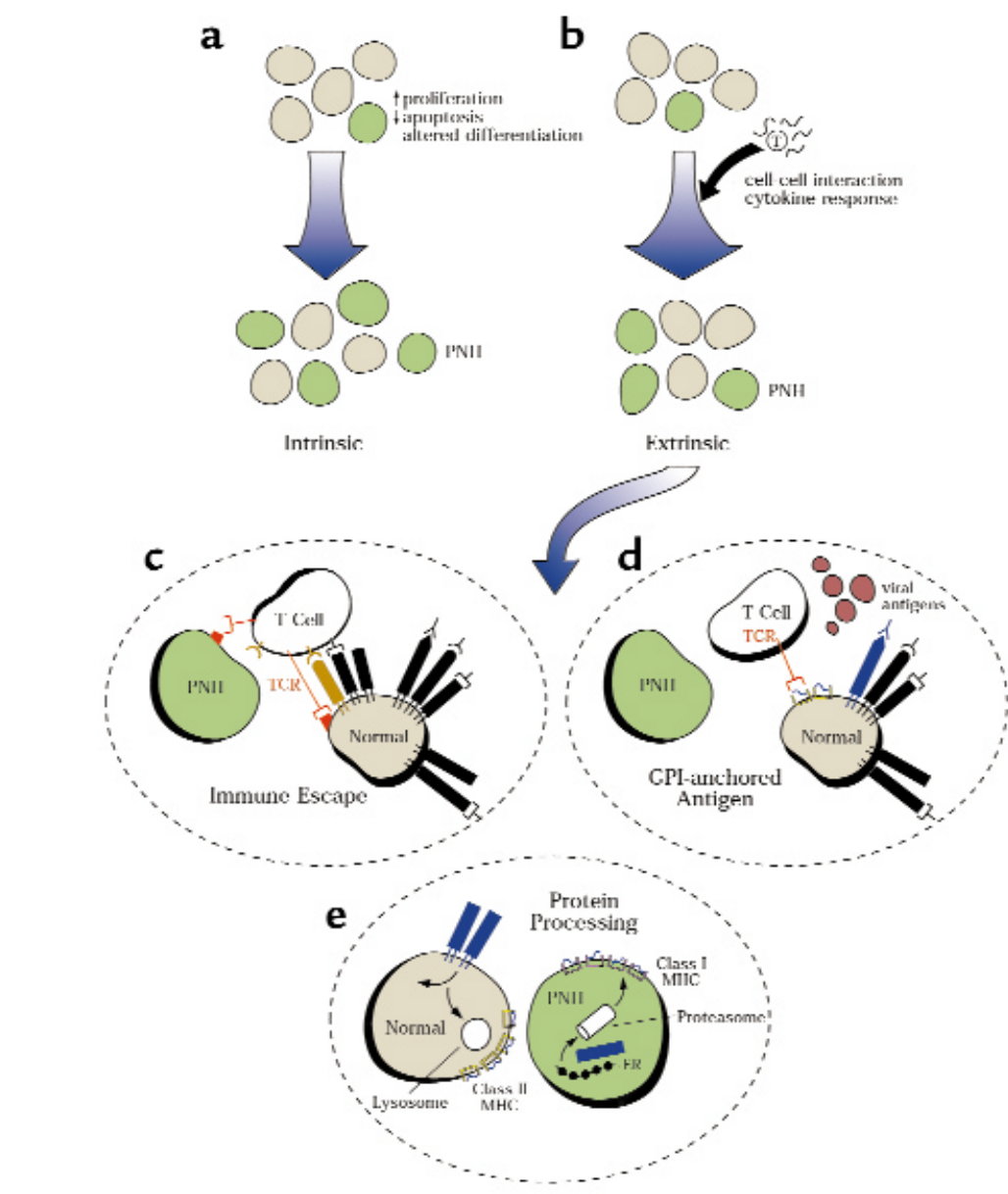

Figure 2

Models for clonal expansion in PNH. (a) In the first model, the PIG-A mutation sufficiently affects cellular function to cause an intrinsic growth advantage. (b) Extrinsic factors are invoked for other models; immune effects are illustrated, but differential responses to hematopoietic growth factors, inhibitory cytokines, or stromal cells are also possible. (c) GPI-anchor deficiency might produce a global deficit in immune recognition, as for example through absence of a costimulatory molecule on the target, providing a general selective advantage to the mutated cells. (d) Alternatively, a GPI-anchor protein might be antigenic, by resemblance to an exogenous antigen or after antigenic spread. (e) Finally, altered protein processing due to the absence of anchor structures might change the nature and quantity of peptides derived from normally GPI-anchored proteins, as well as their MHC presentation. As illustrated, cell surface proteins are normally recycled to the membrane or degraded by lysosomes for class II presentation; in $\mathrm{PNH}$, anchorless proteins likely are processed by the proteasome for class I presentation. Differences in immune response to normal and PNH cells should follow. 
short-lived hematopoietic cells clearly requires further support. Nevertheless, this possibility could help explain the presence of multiple PIG- $A^{-}$clones in PHN individuals, and it appears to be compatible with both the "extrinsic" and "intrinsic" models of PNH shown in Figure 2. Unfortunately, however, it sheds little light on the stability of PIG$A^{+}$and PIG- $A^{-}$cell populations in these subjects. The most direct inference from the current work is that the PNH cells have survived some in vivo injury sustained by the normal progenitors, consistent with the hypothesis of immune escape. That this insult might indeed be immunologic is suggested by the expression of Fas on the normal but not on the GPI-anchor protein-deficient cells and concomitant sensitivity to Fas ligand-mediated apoptosis. Although the authors suggest that this finding supports a resistance to programmed cell death among PNH cells, the result cannot be generalized to other apoptosis inducers. There is no obvious mechanism for altered CD95 expression secondary to GPI-anchored protein deficiency, and again such differences have not been revealed in other immunologic assays. The ability to demonstrate functional differences in inhibition (Fas agonist) and stimulation (Fas antagonist in the absence of an obvious source of Fas ligand) in poorly growing cells is admirable.

Nevertheless, the character of the interaction between a PIG- $A^{-}$clone and either the normal hematopoietic environment or the failed marrow milieu - and indeed of the cause-and-effect sequence of PNH and $\mathrm{AA}$ - remains frustratingly unsettled. Several relationships can be hypothesized and tested (Figure 2, b-e). In the first, a general deficit in effector recognition of a defective cellular target - due, for example, to decreased binding of costimulatory molecules secondary to the GPI-anchor protein deficiency - would explain the emergence of normally quiescent but now resistant $\mathrm{PNH}$ clones under conditions of autoimmune attack (Figure $2 \mathrm{~b}$ ). The data of Chen and colleagues (27) are consistent with this view, but in the context of many contradictory experiments, the effect would necessarily be subtle and manifest only under special in vivo circumstances. In addition, such a dependence of PNH cell viability on immune status is hard to reconcile with the stability of PNH clones over time and despite changes in blood counts and treatment regimens. In the second and third models, in contrast, the mutated cell has a more primary role in the development of bone marrow failure. GPIanchored proteins might serve as antigens, either due to molecular mimicry with exogenous peptides (perhaps derived from a viral protein; Figure 2d) or subsequently as part of a process of antigenic spread. In the third model, the PNH clone itself would induce autoimmunity. As illustrated in Figure 2e, for example, a global alteration in protein trafficking due to absence of GPI anchors is invoked. As a result, GPIanchorless proteins in the PIG-A- cell are processed by proteasomes and their peptides are displayed by class I MHC molecules, in contrast to the normal pathway of membrane-bound protein degradation via the lysosome and class II molecules. This model would provide for both GPI-anchored protein antigenicity (increased or novel peptide presentation by class I MHC) and escape (decreased class II display). Under normal circumstances, the PNH clone would be suppressed. In other, "tolerant" individuals, clonal expansion might occur unimpeded, leading to predominantly hemolytic $\mathrm{PNH}$. In the setting of an aberrant or exaggerated immune response, normal hematopoiesis would suffer, leading to AA. There may be major, unanticipated consequences of failed GPI-anchor synthesis beyond deficient expression of certain membrane proteins.

In PNH, models and experiments will need to account for a number of remaining puzzling questions that are both clinically relevant and of general biologic importance. Biochemically, little is understood of the presumed special function of the GPI anchor in the mammalian cell - in altering the mobility of membrane proteins, in internal signal transduction, in collaboration with other proteins in rafts, in cell-cell protein transfer, and even in shedding from the cell surface, since there is sufficient inactive phospholipase C in plasma to release all GPIanchored proteins from circulating cells. Other questions relate to the dynamics of hematopoietic cells in PNH: Why is there such a tiny proportion of PIG- $A^{-}$progeny in normal individuals? Why do PIG-A- clones persist for years, even decades, supplying the entire blood production of a patient? And why do clones appear to become extinguished in rare individuals? Clini- cally, we need to account not only for the close relationship of a clonal, somatically acquired genetic disorder and immunologically mediated bone marrow failure, but also for evidence of generalized marrow depression in all forms of $\mathrm{PNH}$, the multiplicity of clones in disease, and the inexorable progression to refractory fatal thrombosis or AA in many patients. Continued examination of this rare disease should yield further insights into human hematopoietic cell biology, biochemistry, and immunology.

1. Rosse, W. 2000. A brief history of PNH. In PNH and the GPI-linked proteins. N.S. Young and J. Moss, editors. Academic Press. San Diego, California, USA. 1-20.

2. Young, N.S., and Maciejewski, J. 1997. The pathophysiology of acquired aplastic anemia. N. Engl.J. Med. 336:1365-1372.

3. Frickhofen, N., and Rosenfeld, S.J. 2000 Immunosuppressive treatment of aplastic anemia with antithymocyte globuilin and cyclosporine. Semin. Hematol. 37:56-68.

4. Luzzatto, L., and Nafa, K. 2000. Genetics of PNH. In PNH and the GPI-linked proteins. N.S.Young and J. Moss, editors. Academic Press. San Diego, California, USA. 21-48.

5. Okazaki, I., and Moss, J. 2000. The function of GPI-anchored proteins. In PNH and the GPI-linked proteins. N.S.Young and J. Moss, editors. Academic Press. San Diego, California, USA. 159-177.

6. Schofield, L. 2000. GPI in lower animals. In PNH and the GPI-linked proteins. N.S.Young and J. Moss, editors. Academic Press. San Diego, California, USA. 179-198.

7. Sevlever, D., Chen, R., and Medof, M.E. 2000. Synthesis of the GPI anchor. In PNH and the GPIlinked proteins. N.S.Young and J. Moss, editors. Academic Press. San Diego, California, USA. 199-220.

8. Takeda, J., et al. 1993. Deficiency of the GPl anchor caused by a somatic mutation of the PIGA gene in paroxysmal nocturnal hemoglobinuria. Cell. 73:703-711.

9. Bessler, M., et al. 1994. Paroxysmal nocturnal haemoglobinuria $(\mathrm{PNH})$ is caused by somatic mutations in the PIG-A gene. EMBO J. 13:110-117.

10. Hertenstein, B., et al. 1995. Emergence of CD52-, phosphatidylinositolglycan-anchor-deficient T lymphocytes after in vivo application of Campath$1 \mathrm{H}$ for refractory B-cell non-Hodgkin lymphoma. Blood. 86:1487-1492.

11. Araten, D., Nafa, K., Pakdeesuwan, K., and Luzzatto, L. 1998. PNH cells and PIG-A gene mutations in normal donors. Blood. 92(Suppl. 1):471a. (Abstr.)

12. Kinoshita, T., Bessler, M., and Takeda, J. 2000 Animal models of PNH. In PNH and the GPI-linked proteins. N.S.Young and J. Moss, editors. Academic Press. San Diego, California, USA. 139-158.

13. Dunn, D., et al. 1996. A knock-out model of paroxysmal nocturnal hemoglobinuria: PIG-Ahematopoiesis is reconstituted following intercellular transfer of GPI-anchored proteins. Proc. Natl. Acad. Sci. USA. 93:7938-7943.

14. Kawagoe, K., et al. 1996. Glycosylphosphatidylinositol-anchor-deficient mice: implications for clonal dominance of mutant cells in paroxysmal nocturnal hemoglobinuria. Blood. 87:3600-3606

15. Rotoli, B., Robledo, R., Scarpato, N., and Luzzatto, L. 1984. Two populations of erythroid cell progenitors in paroxysmal nocturnal hemoglobinuria. Blood. 64:847-851. 
16. Okuda, K., et al. 1990. Expression of decay-accelerating factor on hematopoietic progenitors and their progeny cells grown in cultures with fractionated bone marrow cells from normal individuals and patients with paroxysmal nocturnal hemoglobinuria. Exp. Hematol. 18:1132-1136.

17. Maciejewski, J.P., Sloand, E.M., Sato, T., Anderson, S., and Young, N.S. 1997. Impaired hematopoiesis in paroxysmal nocturnal hemoglobinuria/aplastic anemia is not associated with a selective proliferative defect in the glycosylphosphatidylinositol-anchored protein-deficient clone. Blood. 89:1173-1181.

18. Nissen, C., et al. 1999. High incidence of transiently appearing complement-sensitive bone marrow precursor cells in patients with sever aplastic anemia: a possible role of high endogenous IL-2 in their suppression. Acta Haematol. 101:165-172.

19. Ware, R.E., et al. 1998. The PIG-A mutation and absence of glycosylphosphatidylinositol-linked proteins do not confer resistance to apoptosis in paroxysmal nocturnal hemoglobinuria. Blood 92:2541-2550.

20. Young, N.S. 1992. The problem of clonality in aplastic anemia. Dr. Dameshek's riddle, restated. Blood. 79:1385-1392.

21. Luzzatto, L., Bessler, M., and Rotoli, B. 1997. Somatic mutations in paroxysmal nocturnal hemoglobinuria: a blessing in disguise. Cell. 88:1-4.

22. Dunn, D.E., et al. 1999. Paroxysmal nocturnal hemoglobinuria cells in patients with bone marrow failure syndromes. Ann. Intern. Med. 131:401-408.

23. Takami, A., Zeng, W., Wang, H., Matsuda, T., and Nakao, S. 1999. Cytotoxicity against lymphoblastoid cells mediated by a T-cell clone from an aplastic anemia patient: role of CD59 on target cells. Br. J. Haematol. 107:791-796.

24. Dunn, D.E., et al. 1997. The PNH phenotype (GPI-anchored protein-deficiency) protects hematopoietic targets against lymphocytoxic attack. Blood. 90(Suppl. 1):407a. (Abstr.)

25. Asea, A., et al. 2000. HSP70 stimulates cytokine production through a CD14-dependent pathway, demonstrating its dual role as a chaperone and cytokine. Nat. Med. 6:435-442.

26. Hollander, N., Selvaraj, P., and Springer, T.A. 1988 Biosynthesis and function of LFA-3 in human mutant cells deficient in phosphatidylinositolanchored proteins. J. Immunol. 141:4283-4290. 27. Chen, R., et al. 2000. Impaired growth and elevated Fas receptor expression in $\mathrm{PIGA}^{+}$stem cells in primary paroxysmal nocturnal hemoglobinuria. J. Clin. Invest. 106:689-696.

28. Iwamoto, N., et al. 1996. Preferential hematopoiesis by paroxysmal nocturnal hemoglobinuria clone engrafted in SCID mice. Blood. 87:4944-4948.

29. Parker, C.J. 2000. Hemolysis in PNH. In PNH and the GPI-linked proteins. N.S.Young and J. Moss, editors. Academic Press. San Diego, California, USA. 49-100. 